\title{
Development of microbeam technology to expand applications at TIARA
}

\author{
T. Kamiya ${ }^{1}$, T. Satoh ${ }^{1}$, M.Koka ${ }^{1}$, and W.Kada ${ }^{2}$ \\ ${ }^{1}$ Takasaki Advanced Radiation Research Institute, Japan Atomic Energy Agency (JAEA) \\ ${ }^{2}$ Faculty of Science and Technology, Gunma University
}

Abstract

Herein, we review the last half decade of progress in ion-microbeam technology and applications at the Takasaki Ion Accelerators for Advanced Radiation Applications facility. Materials were microanalysed with the light-ion-microbeam system by combining micro-particle-induced $\mathrm{x}$-ray and $\gamma$-ray emission, nuclear-reaction analysis and micro-ion-beam-induced luminescence to analyse elements, including light elements such as lithium, boron or fluoride, and also their chemical states. For microfabrication, we used particle-beam writing and techniques of maskless patterning to processes materials without etching. The goal was to develop optical, magnetic or other new types of microdevices with both light-ion and the heavy-ion microbeam systems. In addition, techniques were developed to monitor in real time every individual ion injection by using an efficient scintillator or a thin diamond particle detector in both heavy-ion and high-energy heavy-ion microbeam systems. 


\section{Introduction}

The history of ion-microbeam technology began in the 1970s with the advent of proton microbeams, which were first used for local elemental analysis at microscopic length scales $[1,2]$. Since then, the applications of this technology have developed remarkably across numerous fields in science and technology, such as biomedicine, materials science, semiconductor-device engineering, geology, environment and archaeology [3-13]. In addition, techniques to focus ion beams have also seen much improvement, to the point that the highest spatial resolution is now on the order of several tens of nanometres [14]. While the majority of ion-microbeam systems focus light ions, such as protons or helium ions, heavy ions can also be used in the same manner in a microbeam. Heavy-ion beams are typically applied to study the effects of radiation on semiconductor devices or in biological cells by using the single-ion-hit technique, which exploits the diversity of the irradiation effects $[15,16]$.

Micro-particle-induced x-ray emission (micro-PIXE) using a heavy-ion microbeam offers the interesting possibility of high energy resolution, as demonstrated by Mokuno et al. who used wavelength-dispersive x-ray spectroscopy to detect chemical effects with high sensitivity [17].

Research and development in ion-microbeam technology and applications at the Takasaki Ion Accelerators for Advanced Radiation Applications (TIARA) facility at the 
Takasaki Advanced Radiation Research Institute of the Japan Atomic Energy Agency has been ongoing since 1990. To expand the variety of applications for ion-beam analysis and to fabricate mesoscopic-scale systems, we have maintained three different types of ion-microbeam systems, which were connected to a $3 \mathrm{MV}$ single-ended accelerator, a $3 \mathrm{MV}$ tandem accelerator and an azimuthally-varying-field cyclotron $(K=$ 110), as summarised in our previous paper [18]. The present paper summarises progress in several technologies, mainly concerning the end stations. This is followed by examples of new applications from the past half-decade.

\section{Microbeam systems at TIARA}

The configuration and performance of the three microbeam systems have not changed significantly since they were installed at TIARA. However, the end stations have been modified to meet the advanced requirements for analysing new samples, fabricating new structures or testing new types of devices or living samples. More applications now deal with environment or life sciences, which have come to be the main fields for such applications, reflecting the demands of ecology, health and safety.

\subsection{Light-ion-microbeam system}

The light-ion-microbeam system was designed for the in-air-micro-PIXE system to 
analyse small biological samples attached to thin polymer films. These films also serve as vacuum windows and initially have a diameter of $1 \mathrm{~mm}[19,20]$. Depending on the application, the target might have to be under vacuum: the beam energy loss in a window material reduces the energy resolution of scanning transmission ion microscopy. However, for three-dimensional elemental analysis, PIXE computed tomography (PIXE-CT) requires that the target be irradiated with a beam that has not traversed a window (a 'windowless' beam). Therefore, for service inside the vacuum, we developed a target stage that includes a rotation stage [21]. Now with the ordinary system, biomedical applications are more practical; for example, the analysis of erythrocyte elements in chronic hepatitis $\mathrm{C}$ patients treated with interferon and ribavirin [22], elemental analysis of lung-tissue particles and intracellular iron content of alveolar macrophages in pulmonary alveolar proteinosis [23] and fluorine analysis of human enamel around fluoride-containing materials under different $\mathrm{pH}$ cycling [24]. This system was also used to develop materials for Li-ion batteries, in particular, to obtain the distribution of $\mathrm{Li}$ in the electrode by means of particle-induced $\gamma$-ray emission (PIGE) and nuclear-reaction analysis (NRA) combined with micro-PIXE [25]. For example, we analysed the positive electrode of a Li-ion battery by positioning a thin film in front of the sample to simulate in situ lithium depth profiling [26]. 
In these types of applications that use PIXE or PIGE, the standard setup allows elemental distributions in the sample to be obtained; however, no chemical information related to those elements can be obtained. Recently, however, Kada et al. have developed a system based on ion luminescence combined with ordinary micro-PIXE that allows the distribution of the chemical state on the sample surface to be measured from the visible-light emission in accordance with elemental distribution [27]. By using ion-luminescence microscopic imaging and spectroscopy (ILUMIS), aerosol particles can be analysed and their origins can be estimated [28]. In addition, the system is also used for proton-beam writing (PBW), which was developed in collaboration with the Shibaura Institute of Technology [29]. For the practical use of PBW, the end-station was equipped with an $\mathrm{X}-\mathrm{Y}$ in vacuo stage driven by ultrasonic motors. The stage-control system was originally designed to manipulate targets with a very high precision to expose areas larger than $10 \mathrm{~mm}^{2}$ by using microbeam scanning. Using this system, Miura et al. attempted to draw large-scale patterns in order to change the optical parameters of a 40-mm-long polymethylmethacrylate sample and obtained a working Mach-Zehnder interferometer thermo-optic switch [30]. In another study, by utilizing localized energy deposition as a thermal load form, Kitamura et al. demonstrated that microbeam irradiation introduces unique morphological changes in fluorinated 
polymers with extremely low thermal conductivity [31-33].

Microbeam patterning irradiation can be performed effectively by monitoring ion luminescence (IL) signal, which indicates change of crystal structure or the chemical state of the target material. By the microbeam irradiation of a diamond film, Kada et al. measured the IL related to graphitisation [34].

\subsection{Heavy-ion microbeam system}

The heavy-ion microbeam system and the single-ion hit technique were established for fundamental studies of single-event phenomena (SEPs) induced when various heavy particles are injected into a semiconductor device with a basic diode structure [35-38]. Recently, charge-transient spectroscopy was performed by using alpha particles from a radiation source and the heavy-ion microbeam for characterising transient charge collection in a device and to evaluate any defects induced in the crystal by the radiation. This technique was used to study the characteristics of silicon carbide (SiC) or diamond, which are wide-band-gap semiconductors, for next-generation highly efficient power devices [39-42]. For these studies, the end station was modified to allow the temperature of the semiconductor specimen to be varied over a wide range from 240 to $350 \mathrm{~K}[43]$. 
Note that ion microbeams can cause changes in the physical properties of materials in minute, clearly localized regions. The degree to which irradiation changes the physical properties, such as the optical or magnetic properties, depends on the linear energy transfer of the beam in the material

\subsection{High-energy heavy-ion microbeam}

On the high-energy heavy-ion microbeam system, accurate beam positioning and the single-ion-hit system were established using the same concept as for the tandem accelerator. A high-energy system was required to study of the effect of long-range ion interactions on semiconductor devices or biological cells under an ambient atmosphere (i.e. with the sample outside of the vacuum system and irradiated through a thin window). For cell irradiation and SEP studies, another collimation system was used, which gives a hit accuracy of over $10 \mu \mathrm{m}$ for each ion [43]. Because maintaining a small spot size is difficult for light ions, such as carbon, because of the larger scattering at the collimator edge as compared with heavy ions, [Remark 3] a special system is required to improve the resolution beyond $10 \mu \mathrm{m}$ without causing scattering. Forming a microbeam requires high-quality beams from accelerators: the intensity must be stable and the beam must have high brightness and a low energy spread. Stability is 
not only required for the total beam intensity $I_{\text {total }}$ but also for its distribution in phase space for the lateral direction $I\left(x, x^{\prime}, y, y^{\prime}\right)$, where $x, x^{\prime}, y, y^{\prime}$ are phase-space coordinates with the origin at the beam centroid. Furthermore, high intensity in the finite region around the origin, which is used to form the microbeam, is required for high brightness. In microbeam focusing, the beam-energy spread $\Delta E / E$ of the accelerator is directly related to the chromatic aberration. Therefore, the accelerator itself is the part of the microbeam technology. Although a cyclotron is suitable to obtain various high-energy heavy-ion beams, it is difficult to obtain such a high-quality ion beam from a cyclotron because it is a radiofrequency accelerator. TIARA undertook some advanced research and development to overcome the problems associated with forming microbeams from cyclotron beams. For example, the techniques of flat-top accelerating and the beam-phase control were developed to confine the beam-energy spread $\Delta E / E$ to a minimum value on the order of $10^{-4}$ [44]. In addition, the beam-phase-bunching effect, which also reduces $\Delta E / E$ of the beam from an AVF cyclotron, was studied briefly both theoretically and experimentally $[45,46]$. Because a cyclotron with external injection has very complicated beam optics in its central region, the beam transport must fit the central region of the cyclotron. For example, Kashiwagi et al. developed a beam-monitoring system to visualise the beam emittance in accordance with the 
acceptance of the system [47].

\subsection{Single-ion-detection system}

In the single-ion-hit technique, developed for both heavy-ion and high-energy heavy-ion microbeam systems, a key problem is how to detect every single ion injected into a sample for studying SEP or cell irradiation. For SEP studies that use a semiconductor-device sample, a single-ion signal can be obtained if an ion injected into the sensitive region generates charges that can be corrected at the device electrode. For biological applications or for nanostructuring by single-ion hits, the sample should be thin enough for the injected ions to penetrate so that the ions may be detected behind the sample. However, if the beam exit window simultaneously serves as the single-ion detector, it is perfect for irradiation of biological cells under ambient conditions. We tried to use thin membranes to detect each single-ion that traversed it; however, such membranes can also act as secondary-electron emitters. Previously, a polycrystalline diamond membrane was tested as a single-ion detector $[48,49]$. Secondary-electron emission was studied to determine if it could serve for ion detection by using an electron multiplier or microchannel plate. Note, however, that unless secondary-electron detectors are placed in a very high vacuum, they suffer easily from noise originating from dark current that results from the residual gas. Maintaining a high vacuum inside 
the end station is usually difficult because of the low evacuation conductance of an end station of a microbeam-lens system. Another solution consists of a high-efficiency scintillation detector; however, this would require perfect darkness around the end station. Conversely, if this problem is clear, a further possibility exists for position-sensitive detection, which would be provided by an additional microscope and high-sensitivity CCD camera system rather than just a photon counter. Along these lines, Satoh et al. tested a system based on a $\mathrm{CaF}_{2}(\mathrm{Eu})$ scintillator and obtained high detection efficiency and position-sensitive accuracy [50]. However, for sufficient efficiency, this method still requires that the scintillator be set behind the sample.

We are now trying to use thin diamond membranes to detect each single ion that traverses it. Diamond has high mechanical strength and is also a semiconductor material, which means that it can serve as a transmission-type particle detector and a vacuum window to extract the ion beam into the air. We showed that a monocrystalline diamond membrane can serve as a high-efficiency transmission-type particle detector; this was performed within an international collaboration with Croatia and France, with the goal being to develop a position-sensitive detector [51,52]. Analysis via the ion-beam-induced charge (IBIC) effect by using a proton beam is also an effective tool to determine the characteristics of semiconductor device $[52,53]$. 


\section{Summary}

For microanalysis of materials, we developed the methods of micro-PIXE, micro-PIGE and micro ion-beam-induced luminescence, which may now be used simultaneously on the light-ion microbeam system at TIARA. These techniques expand the range of possible analyses to include the analysis of the chemical state of elements within a sample. For micro-fabrication, we developed the PBW techniques of maskless patterning on materials without etching, which now constitutes a new tool fabricating microdevices. This research was done not only on the light-ion-microbeam system but also on the heavy-ion-beam system. Furthermore, the single-ion-hit technique was used primarily to study the ion-induced charge response in various semiconductor devices or to analyse the effects irradiation on individual cells. Such an application requires a technique to monitor every individual ion injection in real time, which improves the reliability and efficiency of the ion irradiation. We are also beginning the development of an efficient scintillator and a high-sensitivity camera for studying an additional implementation of a thin-film-type particle detector.

\section{Acknowledgements}


This work was partly supported by Quantum Beam Technology Program of the Ministry of Education, Culture, Sports, Science and Technology (MEXT), Japan, the project 'Development of high-energy ion microbeam technology for novel applications of diamond' under the Strategic Japanese-Croatian Cooperative Program on Materials Science of the Japan Science and Technology Agency and the Ministry of Science, Education and Sports of the Republic of Croatia and Grant-in-Aid for Scientific Research (A), 26249149 MEXT. One of the applications of the heavy-ion microbeam was done in part within the framework of the IAEA CRP F11016 'Utilization of ion accelerators for studying and modelling of radiation induced defects in semiconductors and insulators'. The authors would like to thank Enago (www.enago.jp) for the English language review.

References

[1] JA Cookson, Nucl. Instr and Meth. 165 (1979) 477. 
[2] G.J.F.Legge, Nucl. Instr and Meth.B3 (1984) 561-571.

[3] U.Lindh, Nucl. Instr and Meth.B54 (1991) 160-170.

[4] J.W. McMillan, Nucl. Instr and Meth.B30 (1988) 474-479.

[5] D.N.Jamieson, R.A.Brown, C.G.Ryan, J.S.Williams, Nucl. Instr and Meth.B54 (1991) 1-3.

[6] H.Sekiguchi, T.Nishijima, I.Nashiyama, N.Kobayashi, T.Misawa, S.Yoshida, Nucl. Instr and Meth.B54 (1991) 225-230.

[7] SH.Sie, Nucl. Instr and Meth.B75 (1993) 403-410.

[8] M. Jakšić, L. Kukec, V. Valković, Nucl. Instr and Meth.B77 (1993) 49-51.

[9] C.G. Ryan, W.L. Griffin, Nucl. Instr and Meth.B77 (1993) 381-398.

[10] F.Watt, J.P.Landsberg, Nucl. Instr and Meth.B77 (1993) 249-260.

[11] G.E.Coote, R.W.Gauldie, I.F.West, Nucl. Instr and Meth.B54 (1991) 144-150.

[12]C.P.Swann, Nucl. Instr and Meth.B104 (1995) 576-583. 
[13] X.Li, T.Han, X.Zhu, Y.Li,Y.Zheng, H.Shen, Nucl. Instr and Meth.B308 (2013) $1-5$.

[14] F.Watt, Nucl. Instr and Meth.B130 (1997) 1-8.

[15] C.Fournier, P.Barberet, T.Pouthier, S.Ritter, B.Fischer, K.O.Voss, T.Funayama, N.Hamada, Y.Kobayashi, G.Taucher-Scholz, Radiation Research, 171 (2009) 530-540.

[16] G.Vizkelethy, B.L.Doyle, D.K.Brice, P.E.Dodd, MR.Shaneyfelt, JR.Schwank, Nucl. Instrum. Methods Phys. Res. B231 (2005) 467-475.

[17] Y.Mokuno, Y.Horino, T.Tadic, M.Terasawa, A.Kinomura, A.Chayahara, N.Tsubouchi, Nucl. Instrum. Methods Phys. Res. B150 (1999) 109-113.

[18] T. Kamiya, K.Takano,T.Satoh, Y.Ishii, H.Nishikawa, S.Seki, M.Sugimoto, S.Okumura, M.Fukuda, Nucl. Instrum. Methods Phys. Res. B269 (2011) 2184-2188.

[19] T. Kamiya, T. Suda and R. Tanaka, Nucl. Instr. and Meth. B118 (1995) 447.

[20] K. Ishii, A. Sugimoto, A. Tanaka, T. Satoh, S. Matsuyama, H. Yamazaki, C. Akama, T. Amartivan, H. Endoh, Y. Oishi, H. Yuki, S. Sugihara, M. Satoh, T. Kamiya, T. Sakai, K. Arakawa, M. Saidoh, S. Oikawa, Nucl. Instr. and Meth. B181 (2001) 448. 
[21] T. Satoh, M. Oikawa, T. Kamiya, Nucl. Instr. and Meth. B267 (2009) 2125-2127.

[22] T.Nagamine, S.Tomioka, T.Satoh, M.Koka, T.Kamiya, to be presented in the $14^{\text {th }}$ international conference on nuclear microprobe technology and applications in 2014 (ICNMTA-2014)

[23] Y.Shimizu, S.Matsuzaki, K.Dobashi, N.Yanagitani, T.Satoh, M.Koka, A.Yokoyama, T.Ohkubo, Y.Ishii, T.Kamiya, M.Mori, Respiratory Research, 12 (2011) 88.

[24] H. Komatsu, H. Yamamoto, Y. Matsuda, T. Kijimura, M. Kinugawa, K.Okuyama, M. Nomachi, K. Yasuda, T. Satoh, S. Oikawa, Nucl. Instrum. Methods Phys. Res. B269 (2011) 2274-2277.

[25] K. Mima, R. Gonzalez-Arrabal, H. Azuma, A. Yamazakie, C. Okuda, Y. Ukyo, H.Sawada, K. Fujita, Y. Kato, J.M. Perlado, S. Nakai, Nucl. Instrum. Methods Phys. Res. B290 (2012) 79-84. 
[26] A.Yamazaki, T.Kamiya, T.Satoh, K.Kunioki, Y.Kato, K.Fujita, C.Okuda, H.Sawada, T.Saiyo, R.Gonzarez-Arrabal, JM.Perlado, to be presented in ICNMTA-2014

[27] W. 28, T.Satoh, A.Yokoyama, M.Koka, T.Kamiya, Nucl. Instrum. Methods Phys. Res. B318 (2014) 42-46.

[28] Wataru Kada, T.Satoh, A.Yokoyama, M.Koka, T.Kamiya, Nucl. Instrum. Methods Phys. Res. B306 (2013) 94-98.

[29] T.Kamiya, H.Nishikawa, T.Satoh, J.Haga, M.Oikawa, Y.Ishii, T.Ohkubo, N.Uchiya, Y.Furuta, Appl. Rad. And Isotopes, 67 (2009) 488-491.

[30] K.Miura, H.Kiryu, Y.Ozawa, A. Kubota, Y. Hiratani, O.Hanaizumi, T.Satoh, Y.Ishii, M.Kohka, K.Takano, T.Ohkubo, A.Yamazaki, W.Kada, A.Yokoyama, T.Kamiya, Key Eng. Mat. 596 (2014)134-138.

[31] A.Kitamura (Ogawa), T.Satoh, M.Koka, T.Kobayashi, T.Kamiya, Nucl. Instrum. Methods Phys. Res. B306 (2013) 288-291. 
[32] A.Kitamura (Ogawa), T.Satoh, M.Koka, T.Kobayashi, T.Kamiya, Nucl. Instrum. Methods Phys. Res. B307 (2013) 610-613.

[33] A.Kitamura (Ogawa), T.Satoh, M.Koka, T.Kamiya, T.Kobayashi, Nucl. Instrum. Methods Phys. Res. B314 (2013) 82-85.

[34]Wataru Kada, Akihito Yokoyama, Masashi Koka, Katsuyoshi Takano1, Takahiro Satoh and Tomihiro Kamiya, Jpn. J. Appl. Phys. 51 (2012) 06FB07.

[35] T. Kamiya, N. Utsunomiya, E. Minehara, R. Tanaka and I. Ohdomari, Nucl. Instr. and Meth. B64 (1992) 362.

[36] T. Kamiya, T. Suda and R. Tanaka, Nucl. Instr. and Meth. B118 (1995) 423-425.

[37] S.Onoda, T.Hirao, JS.Laird, T.Okamoto, Y.Koizumi, T.Kamiya, Nucl. Instr. and Meth. B231 (2005) 497-501.

[38] T.Hirao, S.Onoda, M.Oikawa, T,Satoh, T.Kamiya, T.Ohshima, Nucl. Instr. and Meth. B267 (2009) 2216.

[39] N. Iwamoto, B. C. Johnson, N. Hoshino, M. Ito, H. Tsuchida, K. Kojima and T.Ohshima, J. Appl. Phys. 113, (2013) 143714. 
[40] S. Onoda, N. Iwamoto, S. Ono, S. Katakami, M. Arai, K. Kawano, T. Ohshima

'Transient Response of Charge Collection by Single Ion Strike in 4H-SiC MESFETs', IEEE Trans. Nucl. Sci 56, 3218-3222 (2009).

[41] T. Ohshima, S. Onoda, N. Iwamoto, T. Makino, M. Arai, Y. Tanaka, Chapter 16 'Radiation Response of Silicon Carbide Diodes and Transistors' of Physics and Technology of Silicon Carbide Devices, Edited by Y. Hijikata, InTech, ISBN 978-953-51-0917-4, pp.379-402 (2012).

[42] T.Ohshima, N.Iwamoto, M.Koka, S.Onoda, T.Makino, T.Kamiya, W.Kada, Y.Kanbayashi, O.Hanaizumi, N.Hoshino, H.Tsuchida, K.Kojima, to be presented in ICNMTA-2014.

[43] T.Kamiya, T.Hirao, Y.Kobayashi, Nucl. Instr. and Meth. B219-220 (2004) $1010-1014$.

[44] S.Kurashima, K.Yoshida, M.Oikawa, T.Satoh, N.Miyawaki, T.Yuyama, S.Okumura, H.Kashiwagi, I.Ishibori, T.Nara, T.Kamiya, M.Fukuda, W.Yokota, Nucl. Instr and Meth B267 (2009) 2024-2027. 
[45] N.Miyawaki, M.Fukuda, S.Kurashima, H.Kashiwagi, S.Okumura, K.Arakawa, T.Kamiya, Nucl. Instr and Meth A715 (2013) 126-131.

[46] N.Miyawaki, M.Fukuda, S.Kurashima, S.Okumura, H.Kashiwagi, T.Nara, I.Ishibori, K.Yoshida, W.Yokota, Y.Nakamura, K.Arakawa, T.Kamiya, Nucl. Instr and Meth A636 (2011) 41-47.

[47] H. Kashiwagi, N. Miyawaki, S. Kurashima and S. Okumura, Rev. of Sci. Inst. 85 (2014) $02 \mathrm{~A} 735$.

[48] T.Kamiya, M.Cholewa, A.Saint, S.Prawer, G.J. F. Legge, J.E. Butler and D.J.Vestyck Jr, Appl. Phys. Lett. 71 (1997) 1875-1877.

[49] M. Cholewa, T. Kamiya, A. Saint, S. Prawer, G. Legge, J.E. Butler, D.J. Vestyck Jr, Diamond Related Material 7 (1998) 510-512.

[50] T.Satoh, Masahi Koka, Wataru Kada, Akihito Yokoyama, Tomihiro Kamiya, Nucl. Instrum. Methods Phys. Res. B332, (2014) 242-244.

[51] V. Grilj, N. Skukan, M. Pomorski, W. Kada, N. Iwamoto, T. Kamiya4 T. Ohshima and M. Jakšić, Appl. Phys. Lett. 103 (2013) 243106-1-4. 
[52] V. Grilj, N. Skukan, M. Jakšić, W. Kada, T. Kamiya, Nucl. Instrum. Methods Phys. Res. B 306 (2013) 191-194.

[53] 1. W. Kada, T. Kamiya, N. Iwamoto, S. Onoda, V. Grilj, N. Skukan, T. Makino, M. Koka, T. Satoh, M. Jaksic, and T. Ohshima, Transaction of Material Research Society of Japan, 38, (2013) 279-282. 